Øystein Kravdal

Department of Economics

PB 1095 Blindern

N-0317 Oslo

NORWAY

e-mail: okravdal@econ.uio.no

fax: 47-22-855035

\title{
SOCIAL INEQUALITIES IN CANCER SURVIVAL
}

\author{
Øystein Kravdal \\ Department of Economics, University of Oslo \\ and \\ Cancer Registry of Norway
}

RUNNING TITLE: SOCIAL INEQUALITIES IN CANCER SURVIVAL 
ABSTRACT

Social differentials in survival from 12 common types of cancer were assessed by estimating a mixed additive-multiplicative hazard model on the basis of individual register and census data for the whole Norwegian population. The excess all-cause mortality among cancer patients compared with similar persons without a cancer diagnosis was significantly related to education, occupation and income. It was, on the whole, about $15 \%$ lower for men or women who had completed a post-secondary education than for those with only compulsory schooling, taking age, period and registered differences in tumor characteristics and stage at the time of diagnosis into account. The data do not provide clear indications of whether differences in host factors, such as co-morbities and immune functions, or differences in treatment and care are primarily responsible for these inequalities in cancer survival. 
The persistently wide gaps in life expectancy between rich and relatively poor citizens in the industrialized world, even within the supposedly egalitarian Nordic countries, are well documented (e.g., Marmot 1995; Valkonen 1989; Vågerö and Lundberg 1995). Although affluence certainly also takes its toll, it is the material deprivation and various kinds of behaviour prevalent in the lower social classes that - in addition to being important concerns themselves are the most severe threats to a long and healthy life. This situation can be hard to accept from a human rights perspective where equal opportunities in a wide sense is a key ingredient. A reduction of the social differentials in mortality has therefore also been proclaimed by the European region of the World Health Organization (WHO 1985) as one of the major health challenges. Appropriate interventions require, of course, an accurate description of these differentials, as well as a more profound knowledge of their determinants.

In industrialized countries, cardiovascular disease and cancer are now the dominant causes of death, with the former being more than twice as common as the latter. Death rates from cardiovascular diseases have declined in Norway over the last few decades for both sexes, because of both prevention and treatment. Total cancer mortality (i.e., death rates from all types of cancer pooled) for men was on the upturn during the 1970s and 1980s, however. The incidence has, with few exceptions (e.g., stomach cancer), increased considerably, while there have been rather weak improvements in the survival from many of the most common types.

Much smaller social differentials show up in total cancer mortality than in cardiovascular mortality (e.g., Valkonen 1989; Vågerö and Lundberg 1995). However, the differentials are pronounced for some cancer sites, although not always with the poorer individuals displaying the highest mortality (e.g., Harvard Report on Cancer Prevention 1996). Thus, there can be little doubt that the malignant diseases also deserve attention from a social equality perspective. With the intention of learning about the functioning of the health care system and the possible 
gains that can be made, a special focus on cancer may actually be particularly worth while, because this is one of the few diseases that are registered in many countries. This registration provides an opportunity to analyse mortality as a two-step process, the first being the development toward a diagnosed cancer, and the second being the subsequent progression of the disease. The socioeconomic resources may play different roles in these two steps. Whereas wealth may be linked with a high risk of developing cancer - because affluence, for example, may tend to induce some unhealthy dietary habits - the survival rate among the socially advantaged may well be quite high. In fact, such a pattern of social gradients appeared very clearly in a recent register-based study of prostate cancer in Norwegian men (Harvei and Kravdal 1997).

Social determinants of cancer survival have received much attention (although less than those of cancer incidence) since the early studies by, for example, Cohart (1955), who provided a rough description of gross differences between rich and poor regions. (For another example of a quite early, but much more thorough study, see Berg et al.(1977).) In a recent review of several cancer survival studies of fairly mixed quality, it was concluded that the effect of socioeconomic resources is, on the whole, favourable rather than unfavourable (Kogevinas and Porta 1997). Although this may well be true as a rough generalization, the picture must still be considered very blurred, given the widely different estimates that have been produced (without clearly reflecting corresponding differences in statistical methods or, for example, health policy). For many common cancer sites, there is still considerable uncertainty even about the direction of the social gradient in the survival rates. Moreover, very little is known about the reasons for the survival inequalities (e.g., Auvinen and Karjalainen 1997).

In this study, very rich data and a recently developed method are used to produce a more detailed and statistically solid picture of differentials in cancer survival than has been possible in previous studies. Although it is potentially important even as pure description, this improved empirical platform is also meant to shed some additional light on the processes thought to be responsible for survival inequalities. 
The data file covers the entire Norwegian population, and includes more than 100000 persons who had developed cancer in one of the selected sites and died within 5 years of diagnosis (in addition to all those without these diagnoses and those still alive after 5 years). Individual socioeconomic variables have been extracted from the Population Censuses, whereas a majority of the previous studies have been based on aggregate indicators, for example data for the census tract in which the patients live (see criticism by, for example, Greenwald et al. (1994)). Another advantage of this data source is that it includes information on stage and histological type and grade, which have often not been available in other large-scale studies. Controlling for such variables leaves socioeconomic effects that must be the result of other and more interesting factors than the relatively well-established early cancer detection among the socially advantaged.

The statistical method that is used allows a control for social mortality differentials among persons without a cancer diagnosis. As also pointed out by Auvinen et al. (1995), the usual calculations of relative survival without such controls tend to overestimate the social inequalities. The bias is small for cancers that are particularly aggressive, but can reach a considerable size for other sites, for example the prostate, as shown by Kravdal (1997). (In addition to giving a more correct picture of survival patterns, this approach gives, as a by-product, some estimates of differentials in general mortality that are relevant to take into account in part of the discussion.)

As many as 12 common cancers are considered. In such a multi-site study, it is possible to get an impression of the mediating role played by treatment, by comparing social survival gradients for cancers reckoned to respond to treatment with those for cancers that do not. As a more direct check of the treatment effect, a variable describing very roughly the type of primary treatment is included in some models. Such variables have rarely been available in other studies.

It is also a strength of this study that there are three different socioeconomic indicators, namely education, income, and occupation. Previous analyses have been more narrowly focused on one or at most two of these 
variables, or alternatively such variables as hospital payment status or housing tenure. Education has the advantage that it is stable over the adult life course, and that it is defined for both sexes and all age groups, whereas retirees and many women have no income or occupation recorded. In this study, the impact of income and occupation is therefore only addressed for men. The study will reveal whether both education and income have effects, net of each other. Occupational status (e.g., manual versus non-manual work, low- versus high-level non-manual work) probably operates through many of the same factors as income and education, and may thus not be a particularly interesting variable. However, there are a few occupational groups that deserve special attention in light of the explanations suggested for survival inequalities (reviewed below).

The data also allow the inclusion of marital status and place of residence in the statistical models, which facilitates the interpretation of the socioeconomic effects.

\section{A BRIEF REVIEW OF THE CURRENT STATE OF KNOWLEDGE}

\section{Empirical Patterns}

The majority of the survival studies have focused on one particular cancer site, but some have also considered several sites within the same analytical approach. The most prominent example of the latter is perhaps the Finnish study by Auvinen et al. (1995), which was based on individual socioeconomic data for more than 100000 cancer patients, but unfortunately with no information about stage or histological type and grade. A Canadian multi-site study was based on an even larger number of cancer cases (350 000), but only aggregate socioeconomic indicators were available, and there was no control for stage or histology (Mackillop et al. 1997). Also a fairly large Dutch study (about 15000 cases) considered many sites, but only the impact of aggregate indicators could be assessed (Schrijvers et al. 1995a). In a British multi-site study of about the same size as the Dutch one, housing tenure was 
used as a rough indicator of individual socioeconomic resources (Kogevinas et al. 1991).

In total, colorectal cancer and breast cancer have received most attention, with the results largely suggesting beneficial effects of socioeconomic resources. Such effects for colon or rectum have been reported by, for example, Auvinen (1992), Brenner et al. (1991), Chirikos and Horner (1985), and Schrijvers et al. (1995a), and for breast by Ansell et al. (1993), Bassett and Krieger (1986), Gordon et al.(1992), Karjalainen and Pukkala (1990), LeMarchand et al. (1984) and Schrijvers et al (1995b). All these referred studies have controlled for the possibly earlier stage at diagnosis in the higher social classes. On the other hand, a good prognosis for the socially advantaged was not found net of stage in a colorectal study by Dayal et al. (1987), where there were even indications in the opposite direction for colon cancer. Nor did such an effect show up in the models for breast cancer in Kogevinas et al. (1991) and Schrijvers et al. (1995a), and apparently not in a breast cancer study by Ewertz (1994).

Other sites have been less frequently studied. Significant social gradients, with or without control for stage, have been reported for lung (Mackillop et al. 1997; Schrijvers et al. 1995a), prostate (Dayal et al. 1985; Mackillop et al. 1997), bladder (Auvinen et al. 1995; Mackillop et al. 1997), stomach (Auvinen et al. 1995), malignant melanoma (Geller et al. 1996), cervix (Auvinen et al. 1995; Mackillop et al. 1997), uterus (Auvinen et al. 1995; Mackillop et al. 1997; Steinhorn et al. 1986), ovaries (Auvinen et al. 1995), and pancreas (Kogevinas et al. 1991; Mackillop et al. 1997). On the other hand, other authors have not been able to discern any effect, at least not net of stage, for lung (Auvinen et al. 1995; apparently also Bonett et al. 1994), prostate (Kogevinas et al. 1991), stomach (Mackillop et al. 1997; Schrijvers et al. 1995a), ovaries (Mackillop et al. 1997), leukemia (Kogevinas et al. 1991), and cervix (Kogevinas et al. 1991; apparently also Lamont et al. 1993).

\section{$\underline{\text { Suggested explanations }}$}


Three types of factors are crucial for cancer survival (e.g., Auvinen and Karjalainen 1997). First, biologic

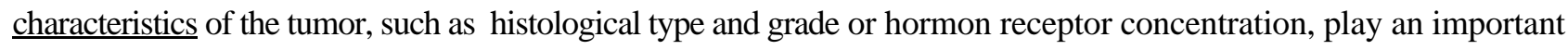
role. Also the degree of metastasis affects the death rates. A late diagnosis, resulting either from "patient's delay" or "doctor's delay", will in some situations have the effect that the tumor has already spread too far to allow efficient treatment. Besides, the timing of diagnosis will also influence the measured survival when there is no treatment that can change the time of death. This is because of the natural development toward steadily higher fatality within the time interval relevant to consider (i.e., when tumors are diagnosed late in the natural development of the disease, the excess death rates caused by this tumor will be higher at any given duration after diagnosis than if it is detected earlier). This is occasionally referred to as a "lead time" bias in survival estimates.

Much of the recent increase in overall 5-year survival rates for some types of cancer is, in fact, the result of earlier detection, partly as a result of screening programs (see, for example, Läärä et al. (1987) for the beneficial impact of cervical cancer screening).

Second, so-called "host factors", such as the existence of co-morbidities, the nutritional status and the immune functions, may influence the progression of the malignancy after diagnosis and the ability to tolerate the treatment. Such characteristics must to a large extent be determined by earlier events, behaviour and processes. For example, Gregorio et al. (1985) showed weak effects of fat intake before diagnosis on survival. However, dietary habits, physical activity, smoking and drinking patterns after diagnosis may also be of some importance. Whether the psychological well-being has any role to play in this, either as a force behind health behaviour or more directly operating through, for example, immune functions, is less certain. It has been suggested that some personality dimensions, such as whether one tends to react to stress by being depressed or aggressive, may influence cancer survival, perhaps through neuro-immune or hormonal mechanisms. Most notably, Spiegel (1990) found that psychosocial treatment had an impact on prognosis. On the whole, however, the scholarly literature suggests that 
coping-style and mood - although important elements of the patients' life quality - have little effect on actual survival (e.g., Buddeberg et al. 1996; Hilakivi-Clarke et al. 1993).

Third, treatment is, of course, a crucial determinant. Survival may depend on the type of primary and followup treatment that is offered, the actual quality of the treatment, as well as the patient's compliance with the treatment.

Education, income and occupation may influence survival through all these three main channels. There is much empirical evidence for a social effect operating through stage. Some authors have found that a relationship between socioeconomic status and survival virtually disappears when it is controlled for stage (e.g., Lamont et al. (1993) for cervical cancer), or that as much as, say, half of it is explained (Auvinen (1992) for colorectal cancer). As is elaborated on in a subsequent section, it is not difficult to imagine also host factors or treatment as responsible for the social gradients in survival, but there is very little empirical evidence for this. One example is the small-scale study by Greenwald et al. (1996), in which it was found that income influenced the survival from non-small-cell lung cancer through a surgery variable. (This accords with Greenberg et al. (1988), who reported less use of surgery as treatment for lung cancer among American patients without private medical insurance, although with no negative impact on their survival.) Similarly, Auvinen (1992) showed that inclusion of a surgery variable eliminated the effect of social status on survival from colorectal cancer in Finland, without interpreting this is as a clear signal of discrimination of the lower social classes. Some authors have also discussed treatment effects by comparing across cancer sites, either on the basis of results from one multi-site study or (less relevant) in a review approach. Moreover, a few studies have reported significant survival differentials net of treatment (e.g., Chirikos and Horner 1985) or have found such differentials within groups known to have received the same initial treatment (e.g., Gordon et al., 1992), which at least indicates that there is more to the story than treatment differentials. (Less convincingly, some authors have simply assumed treatment to be similar across various groups admitted to the same hospital; see review by Auvinen and Karjalainen (1997).) 


\section{DATA AND METHODS}

\section{$\underline{\text { Data }}$}

The analysis is based on individual sociodemographic life histories for all men and women with a Norwegian personal identification number, i.e., all those who have lived in Norway for some time after 1960. These life histories have been extracted from the Norwegian Population Register and the Population Censuses of 1960, 1970 and 1980, and include information about date of death, all changes in residence (migration between municipalities and across the national border), as well as marital status, education, income and occupation at the time of the censuses. These biographies have been linked with data from the Norwegian Cancer Registry, which from 1953 has received information on all cancer cases in the population. This compulsory cancer reporting system is based on pathology and cytology reports, clinical records, and death certificates, and provides information about site, basis for the diagnosis, histologic grade and type, and the stage of the disease at the time of diagnosis. These data have been used in a number of previous studies (e.g., Harvei and Kravdal 1997; Kravdal 1995; and references therein).

Twelve common cancer sites are considered in the analysis. At ages above 40 during the observation period 1960-1991, there were more than 150000 deaths among men or women diagnosed with cancer less than 5 years previously. Of these deaths $76 \%$ deaths were of persons with cancer in one of the 12 selected sites.

\section{$\underline{\text { Statistical model }}$}

For each of these 12 cancer sites, the following mixed additive-multiplicative mortality model is estimated:

$$
\mu=\exp (\mathbf{b x})+y \exp (\mathbf{c x}) \exp (\mathbf{d z})
$$

where $\mu$ is total (all-cause) mortality, $\mathbf{x}$ is a vector of categorical sociodemographic co-variates (including age), $\mathbf{b}$ 
and $\mathbf{c}$ are effect vectors, and $y$ is a cancer-diagnosis indicator that takes the value 1 from the time of diagnosis of that particular cancer (if any) and otherwise is 0 . In other words, the first of the two additive terms captures the mortality variation among persons without this cancer diagnosis, which will be referred to below as the "normal" population. (This "normal" population is, of course, very similar to the total population.) The co-variate vector $\mathbf{z}$ includes various characteristics of the disease or the treatment, which have no counterpart in the "normal" population, and $\mathbf{d}$ is the corresponding effect vector. For a cancer patient with characteristics $\mathbf{x}$, mortality is thus $\exp (\mathbf{c x}) \exp (\mathbf{d z})$ higher than that for a similar individual without such a diagnosis. It is reasonable that this excess mortality is positive (except perhaps after very long durations of particularly severe disease, when the remaining patients may have an extremely strong constitution and a lower mortality than those without cancer who otherwise have the same observed characteristics).

A few models that are not site-specific are also estimated. In these models, y takes the value of 1 from the time of diagnosis of any of the 12 cancers, and a site variable is included in $\mathbf{z}$ (in which case the "normal" population is less similar to the total polulation).

The models are estimated in the AMFIT Poisson-regression module in EPICURE (Preston et al. 1993). As explained in, for example Agresti (1990), Poisson regression is indistinguishable from models for piecewise constant hazard rates where co-variates are categorical. A self-made PASCAL program, operating on the individual-level register and census data, is used to compute the tables of deaths and exposures that are fed into AMFIT.

This method has also been used in a recent prostate cancer study (Harvei and Kravdal, 1997).

\section{Motivation for the model structure}

Mortality studies are often based on a competing-risk framework, with a number of mutually exclusive registered 
causes of death as the possible outcomes. Total mortality is then the sum of various sub-risks, such as the risk of dying with cancer as the registered cause, the risk of dying with heart disease as the registered cause, and the risk of dying from other diseases. In the discussion below, the last two outcomes are denoted as non-cancer deaths. According to Norwegian regulations, a person without a cancer diagnosis can only be registered with a non-cancer death. (If a previously undetected cancer is noticed at the time of death, for example as the result of an autopsy, it will be registered post mortem regardless of whether it is believed to have caused the death.) Among those who do have a cancer diagnosis, death is, of course, very often registered as the result of cancer. In other words, the malignant disease can be considered as producing an extra additive contribution to mortality that otherwise had not been possible. However, it is also likely that patients with a cancer diagnosis may suffer an extra high non-cancer mortality. Unless the patient obviously dies as a result of a progressing malignancy, the role played by this disease for the development towards death may be unknown, and even when the causal mechanisms are thought to be known, deciding on a (main) cause to include on the death certificate may be a matter of judgement. For example, the emotional stress from cancer may increase the chance of being involved in a motor vehicle accident, which may then (correctly) be registered as the cause of death. Alternatively, the patient may die from stroke in connection with an early surgical removal of malignant tissue, or there may be more long-term, harmful side-effects of treatment, both of which may lead to a formally registered non-cancer death. There may also be contributions in the opposite direction, but on the whole it is likely that a malignant disease increases rather than decreases non-cancer mortality.

Consequently, a focus on formally registered cancer deaths will lead to an underestimation of the total impact of the malignancy. Moreover, the estimates of social and other differentials in the impact of cancer will be biased if the pattern in the excess non-cancer mortality is different from the pattern in registered cancer deaths. For these reasons, and because of lack of information on cause-specific mortality in many countries, there is a long tradition in cancer survival studies for considering all-cause mortality. "Relative survival" was suggested several decades ago 
as a potentially fruitful measure of the aggressiveness of the disease (see references in, for example, Buckley (1984)).

It is defined as the ratio of (all-cause) survival for cancer patients to that among persons without such a diagnosis.

This corresponds to considering a malignancy as giving an additive contribution to all-cause mortality. (Referring now to model (1) above, 5-year relative survival $R_{5}$ will simply be $R_{5}=\exp (-5 \exp (\mathbf{c x}) \exp (\mathbf{d z}))$, provided that all co-variates $\mathbf{x}$ and $\mathbf{z}$ are assumed to be constant during the 5-year interval, and that a one-year counting system is used.) The use of an additive rather than a multiplicative basic structure is discussed further in Kravdal (1997).

Estimates of relative survival for various cancer sites by age and sex are routinely produced in several countries (e.g., Cancer Registry 1996). The "normal" survival with which it is compared is typically taken from vital statistics for the total population. Both such descriptive studies and the more analytic ones have typically been based on a comparison with age-, period- and sex-specific "normal" mortality. This is equivalent to a specification of the additive-multiplicative model (1) with only age, period and sex in the $\mathbf{x}$ in the first term. Such an approach cannot be recommended when the focus is on social status or other variables relevant also for the "normal" population. When a relatively high mortality is observed among cancer patients from the lower social classes compared with other persons of the same sex and age and in the same period for the "normal" population, it results partly from the fact that "normal" mortality among these classes is actually higher than the average for this sex, age and period (i.e., patients in the lower social classes would have had a higher mortality than other patients also in the absence of the disease). This necessitates the inclusion of social status also in the $\mathbf{x}$ in the first term. The bias will be particularly large for the less aggressive cancers that do not dominate total mortality strongly, such as prostate cancer, where the education effect is roughly halved when a control for social differentials in "normal" mortality is included (Kravdal 1997). On the other hand, the bias is small for some other sites, as indicated by estimates in Auvinen et al. (1995) and Karjalainen and Pukkala (1990). 


\section{More detailed specifications of the models}

All Norwegian men and women are followed from age 50 (or 40 in models for leukemia, malignant melanoma, and cancer in the breast or female genitals) or, if born before 1910 (or 1920), from 1960. It is censored at age 80 (because of potentially inadequate case ascertainment at higher ages), at the time or emigration, or by the end of 1991, which was the end of the follow-up period. Moreover, individuals are excluded from the analysis (i.e., contribute neither exposure nor deaths) during periods when they lived in another country. In addition, those who did not participate in one of the censuses are excluded during the 10 years after this census. These two restrictions have very little influence on total exposure time.The very few persons with a cancer diagnosed at autopsy are treated as having never received a cancer diagnosis.

The observations are censored 5 years after diagnosis, if any. This interval is commonly used in cancer survival analysis. Very similar social patterns appear in estimates for 2- and 10-year observation intervals. Mortality is assumed to be constant within 5-year age groups, which was experientally found to be a sufficient control for age.

For simplicity, time since diagnosis is not included in the second term of the model. This has no effect on other parameter estimates.

There is no distinction between persons who had previously been diagnosed with another type of cancer and those for whom the cancer in focus was the first one. In the models that are not site-specific (but where site is included as a control variable) only the first cancer diagnosis is considered.

\section{$\underline{\text { Co-variates }}$}


All co-variates are categorical and time-varying. A level for the co-variate is defined for each month during the follow-up period, and refers to the situation at that time (place of residence) or that in the last previous census (education, income, occupaton and marital status).

The categories for education are i) compulsory ii) secondary (10-12 years of schooling), iii) lower-level postsecondary (normally requiring 13-16 years of schooling), and iv) high (defined as a level corresponding to at least a Master's degree, and thus normally requiring 17 or more years of schooling). No distinction was made between 12 or more years of schooling in the 1960 census, so, for the period 1960-1970, those with exactly 12 years are classified as belonging to category iii), whereas category iv) is not used.

Income (in $100 \mathrm{NOK}$ at 1970 prices) is included in the models for men, using seven categories. A combined variable for occupation and education is also included in some models for men, with a categorization as shown in Tables 5-7. Some groups with a particularly high or low general mortality (Borgan and Kristofersen 1986) are selected as separate categories, as also are physicians. Distinction is made between manual and non-manual workers (at different levels) in accordance with standard socioeconomic classification (Album 1988). If no occupation was recorded in the most recent previous census, the occupation in the census before that (if any) is used. There is a separate category for men with no registration of occupation at any previous census.

The $\mathbf{z}$-variable in the model (see equation (1)) includes site (in the few models that are not site-specific), subsite, stage and histology (type and/or grade). For all types of cancer except cervical cancer and leukemia, stage is defined as localized, regional spread (i.e., direct extension to adjacent organs or structures or spread to adjacent lymph nodes), distant spread (i.e., to distant organs or lymph nodes either by direct extension or through discontinuous metastasis), or unknown. Stage is not a meaningful concept for leukemia. In line with definitions for other sites, the following categories are used for cervical cancer: i) 0+I, ii) II, iii) III +IV, and iv) unknown.

The categorization of histology and sub-site (where relevant) differs across cancer site and is explained in 
notes to Table 2 . The intention behind the categorization is to distinguish, at least, between reasonably large histological groups or sub-sites associated with markedly different survival rates. Also primary treatment is included in the $\mathbf{z}$-vector in some models.

\section{RESULTS AND DISCUSSION}

The impact of education

Colorectal cancer in men is selected as an example in Table 1, where all estimates for the first and second additive term are shown. Eductional level is the only socioeconomic variable included in this model, and it turns out to have a significant net effect on the excess mortality (second term).

In Table 2, education effects are also shown for the other 11 cancer sites. Generally, men or women who have completed a secondary education have at least as favourable a prognosis as those with only compulsory schooling. Only a few effect parameters are estimated to be higher than 1, but not significantly so.

(Tables 1 and 2 about here)

Significant effects are found for both sexes for colorectal, bladder and pancreas cancer, whereas significant effects for stomach cancer, lung cancer, malignant melanoma, and leukemia appear only for men. With respect to the reproductive organs, only survival from breast cancer and prostate cancer are significantly associated with educational level.

When one model is estimated for all cancers (but, of course, with cancer site as an additional control variable in the second term of the model), men with post-secondary education (i.e., the two highest categories pooled together) 
have an excess mortality that is $15 \%$ lower than that among men with only compulsory education (not shown). The corresponding estimate for women is $14 \%$. The equivalent of a Master's degree is associated with an advantage of roughly $22 \%$ for both sexes. Differences in this range accord reasonably well with those found in large data sets from Finland for breast cancer $(30 \%$ difference in Karjalainen and Pukkala 1990), colorectal cancer (9\% difference in Auvinen 1992) and other cancers (Auvinen et al. 1995).

The 5-year survival rate for all cancers pooled (not only the 12 selected for this study), and when it is averaged over stage, is about $50 \%$ in Norway (NOU, 1997). This means that the $22 \%$ difference in excess mortality estimated in this study roughly corresponds to a one-and-a-half-year increase in median survival (defined as the duration at which survival of cancer patients has fallen to half of that among similar persons without this diagnosis). Alternatively, one may say that the 5-year survival rate is $58 \%$ instead of $50 \%$.

\section{$\underline{\text { Other measures of social gradients in cancer survival }}$}

Parameter estimates for a model including both income and education are shown in Table 3 for colorectal cancer.

The follow-up in these models is from 1970, because there is no income information from the 1960 census. (This shorter observation period gives sharper education effects.) The education and income effects for other sites are shown in Table 4. Generally, the effects of education are attenuated when income is included, but remain significant to a large extent. Trend effects of income are significant for many sites, even when the group with zero income is excluded.

(Tables 3 and 4 about here) 
A combined occupation and education variable is included in some models. Estimates for both "normal" and excess mortality are shown in Table 5 for colorectal cancer, whereas only those for excess mortality are shown in Table 6, for each of the eight selected cancer sites for men. Finally, a pooled model for all these sites, but with site included as a control variable, is estimated (Table 7). The last model is stratified by educational level, both for practical reasons (software restrictions on the total number of parameters to include) and to facilitate the comparison between some occupational groups within the same educational level.

(Tables 5-7 about here)

The unimportance of marital status or place of residence as mediators

One might suspect socioeconomic differentials in cancer survival to be partly explained by place of residence and marital status. It is a trivial fact that these two variables are linked with social status variables, although in a somewhat ambiguous causality. It also seems plausible that they influence survival. For example, the place of residence may affect the treatment that is given because of the distance to a hospital. Marital status, or more generally the social network, is no less likely to have an impact. Both the "instrumental support" (i.e help in enacting normal role responsibilities) provided by this network and the "socio-emotional support" may, for example, make the patient eat better (Ell et al. 1992), and - along with the "informational support" - improve compliance with treatment. Besides, having a spouse or near friends may also lead to earlier diagnosis, as discussed by, for example, Nayeri et al. (1992). On the other hand, possible disadvantages have also been suggested (e.g., Siegel 1990).

The empirical evidence for an impact of marital status on actual survival is mixed. Some researchers have failed to see an effect for the type of cancer that they have studied (e.g., Ewertz 1994), some have seen clear effects (Krongrad et al. 1996), and some have found effects for only a few of the cancer sites that they have considered 
(Goodwin et al. 1987; Kvikstad et al. 1995).With respect to social network, Waxler-Morrison et al. (1991) found that friendship and work outside the home had an impact on cancer survival, and Ell et al. (1992) found "perceived adequacy of emotional support" to be important, but, on the whole, results appear to be inconclusive (e.g., Blanchard et al. 1995).

Significant effects of marital status appear in the Norwegian data. Typically, the never-married of both sexes face a poor prognosis, whereas differences between married, widowed and divorcees are small. Marital status is obviously not an important mediator (or confounder), because only quite a small change in the education effect can be discerned when marital status is included in the models for colorectal cancer (Table 8) or other cancers (not shown). Also control for place of residence leaves the education effect estimates unchanged (Table 8; results not shown).

(Table 8 about here)

In light of these results, it is assumed in the following discussion that the socioeconomic variables influence survival through economic and cognitive resources, in a wide sense, rather than social network and proximity to good hospitals, although the last two cannot be fully captured by the marital status and region variables included.

The importance of biological characteristics and stage at diagnosis

It is possible that socially advantaged men or women tend to be diagnosed with quite harmless cancers, for example with a high degree of differentiation of the tumor. This might be the result of both more intensive medical checks revealing tumors that otherwise would have remained undetected, or still unknown biological processes connected 
with an upper-class life style. According to these Norwegian data, however, the better prognosis often reported for the higher social classes is not caused by a more favourable histology. The estimates of education effects are almost insensitive to the inclusion of histologic grade and type (not shown), as has also been found by the few other investigators who have had the opportunity to check this (see review by Auvinen and Karjalainen 1997).

Socioeconomic status may also influence the timing of diagnosis (as well as its quality, as addressed below), which in turn may affect the possibilitites for efficient treatment or be linked with mortality through a less interesting "lead time" effect. Most importantly, a late diagnosis could be the result of inadequate self-examination or a failure to recognize that certain symptoms may stem from a malignancy and that it may be favourable to treat malignancies at an early stage. Besides, there may be inadequate access to or quality of professional examination. In Norway, there should be no purely economic reasons for hesitating to consult general practitioners, who are easily available and will refer to specialists whenever needed. However, there may be a delay after the first contact with the health care system, because the patient will often have to undergo several diagnostic checks, some of which may require considerable waiting-time. A more extreme reason for a delayed diagnosis would be that a cancer is overlooked by mistake at first consultation and not diagnosed until the symptoms have become clearer. Money may not help much in these situations, but those who are insistent, know their way around or have an ability to present their case in a convincing manner may have some advantages.

In support of this argument, the Norwegian data reveal that stage has some importance as a mediator. The earlier diagnosis among the socially advantaged explains a fraction, although a modest one, of their relatively good prognosis. For colorectal cancer, the parameters for the two highest educational groups are 0.84 and 0.81 without a control for stage (not shown), as opposed to 0.87 and 0.83 with such a control included (Table 1 ). In other words, only $2-3 \%$ of the beneficial effect of roughy $17 \%$ is explained by an earlier stage. On the other hand, the beneficial effect associated with the two highest educational levels (pooled) is reduced from $32 \%$ to $24 \%$ when stage is included 
in the breast cancer models. The corresponding percentages for cervical cancer are $27 \%$ and $9 \%$, for uterus cancer $23 \%$ and $11 \%$, for ovarial cancer $14 \%$ and $9 \%$, and for malignant melanoma in women $35 \%$ and $23 \%$. In fact, without control for stage, the effects for post-secondary education would become significant at the $5 \%$ level for all the genital cancers and malignant melanoma.

One cannot possibly expect the information in the cancer registration system to be complete. For example, there may be some differences in the spread of the tumor within each recorded stage. This is partly the result of misclassification. Some patients who receive a particularly thorough check may, for example, be diagnosed with a regional spread that among less intensively diagnosed persons would have been recorded as localized (as discussed from a different perspective by Feinstein et al. 1985). If a more thorough examination has this consequence (rather than the opposite, which cannot be ruled out), patients undergoing such examination, who are perhaps overrepresented among the better educated, would tend to have relatively benign localized cancers as well as relatively benign regional ones. However, as stage on the whole plays a quite modest role as a mediator, this kind of residual stage effect is not taken into account when social differentials in survival net of recorded stage are discussed further below.

Interpreting the main social gradients that appear net of stage: The plausibility of the host-factor and treatment $\underline{\text { explanations }}$

The picture is quite consistent: Even with control for stage, both a high education and a high income enhance survival. Similarly, the prognosis is better for men in non-manual than for those in non-manual occupations., and better in high- than in low-level non-manual occupations (net of education).

As mentioned above, one possible explanation is that host factors are mediators. The impact of such factors 
on survival cannot be checked with these data, but it is at least theoretically plausible that the cognitive resources associated with education, for various reasons not to be elaborated on here, may have a bearing on the general health at the time of diagnosis (or more specific host factors). Also income (or the economic strength, which is determined also by transfers, taxes and assets) may be linked with general health, not only because of the economic opportunities to achieve, for example, a good nutritional status and a favourable physical environment, but also because a high income (net of education) is a signal of cognitive capacity. A similar argument is relevant for occupational status.

Such an association between general health and education, income and occupational status is also suggested in the data. For example, "normal" mortality among men with a few years of education beyond secondary school is $25 \%$ lower than that among men with only compulsory schooling (Table 1). The corresponding figure for women is $28 \%$ (not shown). Similar effects are found for income (Table 3) and occupational status (Table 5).

On the other hand, it is no less plausible that treatment differentials are responsible for the social gradients in survival. Few costs should be of any concern for cancer patients, not least as private clinics offering their often unsubsidized services (which would typically be diagnostic anyway) were much less prevalent during the period under study than today. However, such factors as knowledge, ability to take personal initiative and ability to communicate efficiently, which may captured even by the income variable, are likely to count.

First, there may be differences in the quality of the diagnosis, which is crucial for the treatment. There is, of course, always the possibility that the work in the pathology laboratory is more carefully done when the patient is a well-known person or perhaps a colleague, but, generally, mistakes are likely to be quite random. Those who realize the importance of the diagnosis and ask for a new check or seek a second opinion would then be the advantaged group.

Second, there may be fatal differences in the type of treatment that is chosen, given the diagnosis. It is possible that patients with more socioeconomic resources are less often victim of sloppy work or incompetence. More 
importantly, surgery, radiotherapy and chemotherapy involve a great deal of medical judgement, and individual adaptations may be called for in order to take into account the patients' own view of their health and their ability to tolerate, say, the side-effects of the treatment. In this situation, being generally well-informed and able to describe needs and desires may be helpful.

Third, the treatment that is decided on may not be implemented as planned because of lack of capacity. In Norway, one may in some situations have to wait a few weeks for primary treatment, even when it is supposed to be curative. In addition, various kinds of palliative treatment, which may reduce death rates, are in under-supply. (For specific examples about capacity problems and lack of equipment, see NOU (1997).) Given such constraints, the individual characteristics suggested above as linked to education, income and occupational status could easily have an influence on survival.

Fourth, the quality of the treatment that is given eventually may be inferior because of personal mistakes or mal-functioning equipment. Whether this is likely to happen less often among the richer and better educated in Norway is an open question.

Finally, patients from the highest social classes may possibly follow instructions more accurately, and take more initiative to further consultation or treatment if they notice signs of recurrence or other problems during periods when they are under less close medical surveillance.

\section{A rough empirical check of the relevance of the treatment explanation}

One may get an idea of the importance of the treatment explanation by comparing the differences in survival patterns across cancer sites. Unfortunately, one cannot easily identify sites for which inadequacies in treatment are particularly fatal and closely linked with socioeconomic resources, but it may be assumed, for simplicity, that treatment-induced 
social gradients are most likely to show up for cancers that are generally reckoned to respond reasonably well to treatment.

No good treatment exists yet for stomach, pancreas and (especially small-cell) lung cancer. For the first of these sites, education effects are relatively weak, although significance is attained for men (Table 2). This accords well with some other large-scale studies, in which negligible effects have been reported for stomach cancer. For pancreas cancer, however, significant effects are found for both sexes, and previous studies also point largely in this direction. The effects are less clear for lung cancer. Whereas effects for men are significant, non-significant effects in the opposite direction are estimated for women, as the only exception from the general pattern.

Conversely, quite efficient treatment has been developed for breast, cervical and colorectal cancer (when diagnosed at an early stage), and partly also for bladder cancer, but without particularly clear education effects appearing in the tables. Effects for cervical cancer are not even significant, net of stage.

As mentioned above, little has been done in the past to test the treatment explanation. In a recent review, Kogevinas and Porta (1997) claimed that the evidence for social gradients was strongest for breast and colorectal cancer, which they considered to support the treatment explanation. One must bear in mind, however, that many other sites have received less attention, and that data quality and methods differ markedly between all the studies (which are even from different countries). One single statistically solid multi-site study, such as this study, is a better basis for such comparisons across sites.

Generally, treatment is most efficient among patients with a localized tumor, and, if there were social differences in the treatment, one would therefore expect education effects to be most pronounced in models restricted to the localized cases. In contrast to some other studies (e.g., Karjalainen and Pukkala 1990), there are only weak indications in this direction (not shown).

As a second check of the relevance of the treatment explanation, a treatment variable is included in the 
regression model. As shown in Table 8, this has no impact on the estimated effects of educational level for colorectal cancer, and it also turns out to have very little impact for other cancer sites (not shown). However, this is surely not a good check, because the variable only includes quite rough information about the type of primary treatment and captures nothing about its quality or about the follow-up (palliative) treatment. Moreover, the causality is not altogether clear: Patients in the higher social classes may be selected relatively often for surgery because of their good health, rather than as a result of their socioeconomic resources, and would therefore perhaps have had better survival also without the operation.

\section{Challenging patterns for a few particular occupations}

The results for a few occupational groups are particularly challenging. One of these groups are the physicians, who have a higher annual income than other men with high education. However, their "normal" mortality is not particularly low, which is in accordance with a Finnish study (Rimpelä et al. 1987). Their good cancer prognosis relative to other men with high education, according to indications from site-specific models in Table 6 and the significant effect in Table 7, is therefore not particularly likely to be caused by host factors, whereas a treatment advantage seems more plausible: Above all, physicians have good knowledge about treatment alternatives and about symptoms that call for intervention, and they have easy access to expert opinions in these matters.

Both teachers and men without any recorded occupation are likely to have certain health characteristics that are markedly different from others on the same educational level. At least, their general mortality (Table 5) is relatively low and high, respectively. As shown in Table 7, similar differences are found in survival (significant for men without occupation; weaker indications for teachers), which may thus be interpreted as host-factor effects. However, treatment effects are also plausible, especially for men without an occupation, who may have fewer useful 
acquaintances and certain characteristics that may make them less powerful in their communication with health professionals. It seems more far-fetched to expect teachers to receive better treatment than others on the same educational level (and in high-level non-manual occupations).

Two occupational groups that are even more interesting are the farmers and those who work in hotels and restaurants, as ship officers or as deck and engine-room crew. As shown in Table 5, and known also from other studies (e.g., Borgan and Kristofersen 1986), the former group has a very low general mortality, whereas the latter has a very high one. However, a similar pattern does not appear in cancer survival (the farmers even had a significantly worse prognosis than manual workers), as one would expect if general mortality were a signal of host factors that are important, and under the assumption that treatment differentials in these cases would be small or at least not work in the opposite direction.

In general, but perhaps with less relevance for these two groups, such discrepancies may reflect that some factors responsible for a very high or low "normal" mortality (e.g., a tendency to be involved in hazardous activities) have no importance for the progression of the malignancy. It is also possible that selection, without generally being an important problem, influences the estimates of some social differentials for some of the cancers considered in this study: The host factors that are important for the development after diagnosis may vary with social status for cancer patients in another way than for other men. For example, the generally higher mortality for hotel and restaurant workers than for the farmers may result partly from smoking. Among those who have already developed lung cancer or other smoke-related cancers, however, such differentials in smoking are less pronounced, because the farmers are also likely to have smoked to a very large extent, although not necessarily as much. Therefore, if it is assumed that smoking is also a crucial host factor for the progression of the disease, the excess mortality resulting from cancer may not be as large among hotel and restaurant workers (compared with others) as one might expect from the generally high mortality in this group. Besides, the measure of excess mortality in this study will include more than 
the excess mortality that is actually a causal result of cancer (given that the cancer patients are heavier smokers than others in the same social status group and that smoking is also important for "normal" mortality). The mortality that the patients would have had in the absence of cancer, and with which it should be compared in order to assess the causal effect of the disease, is higher than the "normal" mortality used in this study. This bias will be most pronounced for the group that generally has the lowest smoking rate, i.e., the farmers in this case. To summarize, the relatively high excess mortality for the farmers that is estimated is not necessarily inconsistent with an idea that the generally lower mortality of this group compared with hotel and restaurant workers is caused partly by smoking and that smoking is also an important host factor in the progression of cancer after diagnosis.

\section{CONCLUSION}

This study has demonstrated fairly large differences in cancer survival between various social groups in a country that is reckoned to be an advanced welfare state. The pattern appears for most of the 12 common cancer sites that have been considered in the analysis, including sites for which the empirical evidence has so far been inconclusive. This gradient is not only the result of earlier diagnosis of some cancer types among the socially advantaged, which itself is a challenging phenomenon. Significant effects appear even with controls for stage. By and large, it also seems that the social differentials have remained quite constant during the few decades under study (not shown).

Adding a year or more to the life of an average cancer patient, which would roughly be the result if everyone had the same prognosis as the better educated, would certainly be considered an important step forward, even though it would contribute little to the average life expectancy at birth. Although it is important merely to document such a difference in survival, more needs to be known about the underlying mechanisms before policy conclusions can be drawn. The data provide few clear indications that treatment inequalities are a key intermediate factor, but this 
possibility cannot be rejected either. Such inequalities would be particularly provocative from a health justice perspective and would call for an expansion of the medical services for the less privileged, preferably without making the situation worse for others. Moreover, if treatment inequalities are widely suspected, cancer patients may feel more of a personal responsibility for their situation and may put up a fight to attract resources. Given the importance of this issue, one should soon check more thoroughly whether patients from the lower social classes actually receive less adequate treatment and care than others, with consequences even for their survival.

The idea that the better prognosis in the higher social classes may be caused by a stronger constitution at the time of diagnosis, or more favourable health behaviour afterwards, is perhaps less interesting from a health policy perspective. Support for this host-factor explanation would suggest that it might be worth while to try to strengthen patients' general health - provided that such interventions after diagnosis are not too expensive and are of importance given the health status at the outset. Documentation of such a causal pathway would also underscore the need to take dependency between diseases into account in demographic studies of possible developments in longevity. If the population adopt a generally healthier life style or if some structural barriers to a good health are demolished, we may perhaps witness an improved cancer survival even in the unlikely situation of no progress in treatment. The data do not allow a good check of the relevance of the host-factor explanation. Many results appear to be consistent with such an explanation, but may just as well be taken as support for the treatment explanation.

\section{ACKNOWLEDGEMENTS}

The helpful comments from Tor Haldorsen, Sverre Harvei, Steinar Tretli and other epidemiologists in the Cancer Registry, the very competent assistance from the computer staff, and Elisabeth Parr's and Mariola Gruszozynska's conscientious copying of all the literature that had to be read are greatly appreciated. Thanks are also due to Statistics Norway for allowing the use of the data. The editor and reviewers have given very valuable advice. 


\section{LITERATURE}

Agresti, A. 1990. Categorical Data Analysis. New York: Wiley and sons.

Album, D. 1988. “Kommentarer til standard for inndeling etter sosioøkonomisk status”, Reports 88/18, Statistics Norway, Oslo-Kongsvinger.

Ansell, D., S. Whitman, R. Lipton, and R. Cooper. 1993. "Race, income, and survival from breast cancer at two public hospitals", Cancer 72: 2974-8.

Auvinen, A. 1992. "Social class and colon cancer survival in Finland”, Cancer 70: 402-7.

Auvinen, A., S. Karjalainen, and E. Pukkala. 1995. "Social class and cancer patient survival in Finland”, $\underline{\text { American Journal }}$ of Epidemiology 142: 1089-1102.

Auvinen, A. and S. Karjalainen. 1997. "Possible explanations for social class differences in cancer patient survival”, in M. Kogevinas, N. Pearce, M. Susser and P. Bofetta (eds.), Social Class and Cancer. Lyon: International Agency for Research on Cancer, Scientific Publication 138, 377-97.

Bassett, M.T. and N. Krieger. 1986. "Social class and black-white differences in breast cancer survival”, $\underline{\text { American Journal }}$ of Public Health 76: 1400-3.

Berg, J.W., R. Ross, and H. Latourette. 1977. "Economic status and survival of cancer patients”, Cancer 39: 467-77.

Blanchard, C.G., T.L. Albrecht, J.C. Ruckdeschel, C.H. Grant, and R.M. Hemmick. 1995. “The role of social support in adaptation to cancer and survival”, Journal of Psychosocial Oncology 13: 75-95.

Bonett, A., D. Roder, and A. Esterman. 1984. "Determinants of case survival for cancers of the lung, colon, breast and cervic in South Australia”, The Medical Journal of Australia 141: 705-9. 
Borgan, J.-K. and L.B. Kristofersen. 1986. “Dødelighet i yrker og sosioøkonomiske grupper 1970-1980”, Statistiske analyser 56, Statistics Norway, Oslo-Kongsvinger.

Brenner, H., A. Mielck, R. Klein, and H. Ziegler. 1991. "The role of socioeconomic factors in the survival of patients with colorectal cancer in Saarland/Germany", Journal of Clinical Epidemiology 44: 807-15.

Buckley, J.D. 1984. “Additive and multiplicative models for relative survival rates”, Biometrics 40: 51-62.

Buddeberg, C., M. Sieber, C. Wolf, C. Landolt-Ritter, D. Richter, and R.Steiner. 1996. “Are coping strategies related to disease outcome in early breast cancer?", Journal of Psychosomatic Research 40: 255-64.

Cancer Registry. 1996. Cancer in Norway 1993. Oslo

Chirikos, T.N. and R.D.Horner. 1985. "Economic status and survivorship in digestive system cancers", Cancer 56: $210-7$.

Cohart, E. 1955. "Socioeconomic distribution of cancer of the female sex organs in New Haven”, Cancer 8: 34-41.

Dayal, H.H., L. Polissar, and S. Dahlberg. 1985. "Race, socioeconomic status, and other prognostic factors for survival from prostate cancer", Journal of the National Cancer Institute 74: 1001-6.

Dayal, H.H., L. Polissar, C.Y. Yang, and S. Dahlberg. 1987. "Race, socio-economic status, and other prognostic factors for survival from colo-rectal cancer", Journal of Chronic Diseases 40: 857-64.

Ell, K., R. Nishimoto, L. Mediansky, J. Mantell, and M. Hamovitch. 1992. "Social relations, social support and survival among patients with cancer", Journal of Psychosomatic Research 36: 531-41.

Ewertz, M. 1993. "Breast cancer in Denmark. Incidence, risk factors, and characteristics of survival”, Acta Oncologica 32 : 595-615.

Feinstein, A.R., D.M. Sosin, and C.K. Wells. 1985. "The Will Rogers phenomenon. Stage migration and new diagnostic 
techniques as a source of misleading statistics for cancer survival”, New England Journal of Medicine 312: 1604-1608.

Geller, A.C., D.R. Miller, R.A. Lew, R.W. Clapp, M.B. Wenneker, and H.K. Koh. 1996. “Cutaneous melanoma mortality among the socioeconomically disadvantaged in Massachusetts”, American Journal of Public Health 86 (4):538-44.

Goodwin, J.S., W.C. Hunt, C.R. Key, and J.M. Samet. 1987. “The effect of marital status on stage, treatment, and survival of cancer patients", Journal of the American Medical Association 258: 3125-30.

Gordon, N.H., J.P. Crowed, D.J. Brumberg, and N.A. Berger. 1992. "Socioeconomic factors and race in breast cancer recurrence and survival", American Journal of Epidemiology 135: 609-618.

Greenberg E.R., C.G. Chute, T. Stukel, J.A. Baron, D.H. Freeman, J. Yates, and R. Karson. 1988. "Social and economic factors in the choice of lung cancer treatments: a population based study in two rural states", New England Journal of Medicine 318: 612-7.

Greenwald, H.P., N.L. Polissar, E.F. Borgatta, and R. McCorkle. 1994. "Detecting survival effects of socioeconomic status: problems in the use of aggregate measures", Journal of Clinical Epidemiology 47: 903-9.

Greenwald, H.P., E.F. Borgatta, R. McCorkle, and N. Polissar. 1996. "Explaining reduced cancer survival among the disadvantaged", Milbank Quarterly 74: 215-38.

Gregorio, D.I., L.J. Emrich, S. Graham, J.R. Marshall, and T. Nemoto. 1985. "Dietary fat consumption and survival among women with breast cancer", Journal of the National Cancer Institute 75: 37-41.

Harvard Report on Cancer Prevention 1996. Socioeconomic status. Cancer Causes and Control, 7, suppl 1: S33-5.

Harvei, S. and Ø. Kravdal. 1997. "The importance of marital and socioeconomic status in incidence and survival of prostate cancer", Preventive Medicine 26: 623-32.

Hilakivi-Clarke, L., J. Rowland, R. Clarke, and M.E. Lippman. 1993. "Psychosocial factors in the development and 
progression of breast cancer", Breast Cancer Research and Treatment 29: 141-60.

Karjalainen, S. and E. Pukkala. 1990. "Social class as a prognostic factor in breast cancer survival”, Cancer 66: 819-26.

Kogevinas, M. and M. Porta. 1997. “Cancer survival”, in M. Kogevinas, N. Pearce, M. Susser and P. Bofetta (eds.), Social Class and Cancer. Lyon: International Agency for Research on Cancer, Scientific Publication 138, 177-206.

Kogevinas, M., M.G. Marmot, A.J. Fox, and P.O. Goldblatt. 1991. "Socioeconomic differences in cancer survival”, Journal of Epidemiology and Community Health 45: 216-9.

Kravdal, Ø. 1995. "Is the relationship between childbearing and cancer incidence due to biology or lifestyle? Examples of the importance of using data on men", International Journal of Epidemiology 24: 477-484.

Kravdal, Ø. 1997. “The attractiveness of an additive hazard model: An example from medical demography”, European Journal of Population 13: 33-47.

Krongrad, A., H. Lai, M.A. Burke, K. Goodkin, and S. Lai. 1996. "Marriage and mortality in prostate cancer”, The Journal of Urology 156: 1696-1700.

Kvikstad, A., L.J. Vatten, and S. Tretli. 1995. "Widowhood and divorce in relation to overall survival among middle-aged Norwegian women with cancer", British Journal of Cancer 71: 1343-7.

Läärä, E., N.E. Day, and M. Hakama. 1987. "Trends in mortality from cervical cancer in the Nordic countries: Association with organised screening programmes", Lancet 8544: 1247-9.

Lamont, D.W., R.P. Symonds, M.M. Brodie, N.J. Nwabinelli, and C.R. Gillis. 1993. “Age, socio-economic status and survival from cancer of cervix in the West of Scotland 1980-87”, British Journal of Cancer 67: 351-7.

LeMarchand, L., L.N. Kolonel, and A.M.Y. Nomura. 1984. "Relationship of ethnicity and other prognostic factors in breast cancer survival patterns in Hawaii”, Journal of the National Cancer Institute 73: 1259-65. 
Mackillop, W.J., J.Zhang-Salomons, P.A. Groom, L. Paszat, and E. Holowatny. 1997.“Socioeconomic status and cancer survival in Ontario", Journal of Clinical Oncology 15: 1680-89.

Marmot, M. 1995. "Social differentials in mortality: The Whitehall studies”, in A. Lopez, G.Caselli and T. Valkonen (eds.), Adult Mortality in Developed Countries: From Description to Explanation. Oxford: Clarendon Press, 243-60.

Nayeri, K., G. Pitaro, and J.G. Feldman. 1992. “Marital status and stage at diagnosis in cancer”, New York State Journal of Medicine 92: 8-11.

NOU (1997). “Omsorg og kunnskap!” Norges offentlige utredninger 1997:20. Statens forvaltningstjeneste. Oslo.

Preston D.L., Rubin J.H., Pierce P.A., and McConney M.E. 1993. “Epicure user's guide”. Hirosoft International Corporation, Seattle.

Rimpelä, A.M., M.M. Nurminen, P.O. Pulkkinen, M.K. Rimpelä, and T. Valkonen. 1987. "Mortality of doctors: Do doctors benefit from their medical knowledge?", Lancet 8523: 84-87.

Schrijvers, C.T.M., J.-W.W. Coebergh, L.H. van der Heiden, and J.P. Mackenbach. 1995a.“Socioeconomic variation in cancer survival in the southeastern Netherlands, 1980-89”, Cancer 75: 2946-53.

Schrijvers, C.T.M., J.P. Mackenbach, J.-M. Lutz, M.J. Quinn, and M.P. Coleman. 1995b."Deprivation and survival from breast cancer”, British Journal of Cancer 72: 738-743.

Siegel, K. 1990. "Psychosocial oncology research", Social Work in Health Care 15: 21-43.

Spiegel, D. 1990. “Can psyhoterapy prolong cancer survival?”, Psychosomatics 31: 361-366.

Steinhorn, S.C., M.H. Myers, B.F. Hankey, and V.F. Pelham. 1986. "Factors associated with survival differences between black women and white women with cancer of the uterine curpus", American Journal of Epidemiology 124: 85-93. 
Valkonen, T. 1989. “Adult mortality and level of education: a comparison of six countries”, in J. Fox (ed.), $\underline{\text { Health }}$ Inequalities in European Countries. Aldershot: Gower, 142-162.

Vågerö, D. and O. Lundberg. 1995. “Socioeconomic mortality differentials among adults in Sweden”, in A. Lopez, G.

Caselli and T. Valkonen (eds.), Adult Mortality in Developed Countries: From Description to Explanation. Oxford:

Clarendon Press, 223-242.

WHO. 1985. "Targets for health for all year 2000”, WHO, Regional Office for Europe, Copenhagen.

Waxler-Morrison, N., T. Gregory Hislop, B. Mears and L. Kan. 1991. "Effects of social relationships on survival for women with breast cancer: A prospective study”, Social Science and Medicine 33 (2): 177-83. 
